\title{
Beyond Mucosal Infection: a Role for C. albicans-Streptococcal Interactions in the Pathogenesis of Dental Caries
}

\author{
Megan L. Falsetta $\cdot$ Hyun Koo
}

Published online: 12 January 2014

(C) Springer International Publishing AG 2014

\begin{abstract}
The human body is home to countless microorganisms that can modulate the transition between health and disease. When conditions in the host favor the growth of pathogens, the populations can shift toward their dominance and/or cause disbyosis. In the mouth, commensal organisms are typically most abundant, but when oral hygiene is neglected and sugar is frequently consumed and/or there is a disruption in saliva production/flow, organisms associated with dental caries disease eg, Streptococcus mutans (S. mutans) become more prevalent. These organisms interact with dietary sugars and host saliva to form complex 3-dimensional biofilms on pellicle-coated teeth. The production of exopolysaccharides (eg, via $S$. mutans-sucrose interactions) modulates the assembly of the biofilm matrix, while acid production (eg, by acidogenic flora) and low $\mathrm{pH}$ within the biofilm facilitate the demineralization of the adjacent tooth enamel. During the assembly of cariogenic biofilms, S. mutans likely interacts and competes with other oral microbial species in the mouth. Although it is widely recognized that bacterialfungal interactions commonly occur on mucosal surfaces, their possible role in dental caries has received limited attention. This review provides evidence that interactions between S. mutans and Candida albicans (C. albicans) may be involved in the pathogenesis of early childhood caries.
\end{abstract}

\section{L. Falsetta $(\square)$}

Department of Environmental Sciences, The University of Rochester, 601 Elmwood Ave., Box 850, Rochester, NY 14642, USA

e-mail: megan_wood@urmc.rochester.edu

H. Koo

Biofilm Research Laboratory, Levy Center for Oral Health Research, and Department of Orthodontics, School of Dental Medicine, University of Pennsylvania, Philadelphia, PA 19104, USA
Keywords S. mutans · C. albicans · Glucosyltransferase · Exopolysaccharides $\cdot$ Extracellular matrix $\cdot$ Polymicrobial · Biofilm $\cdot$ Mucosal infection $\cdot$ Dental caries

\section{Introduction}

At many sites within the human body, a precarious equilibrium exists between the microorganisms present, host factors, and the changes occurring in the local environment. In the mouth, the disease dental caries results from complex interactions that occur on pellicle-coated tooth surfaces between (cariogenic) organisms, the products produced by these organisms, host salivary constituents, and dietary carbohydrates [1-4]. Although S. mutans is not always the most abundant species in the mouth, it can rapidly orchestrate the formation of biofilms on teeth when environmental conditions favor the assembly of an extracellular matrix (eg, when sucrose, a substrate for exopolysaccharide production, is frequently available) $[1-5,6 \bullet]$. During the course of cariogenic biofilm development, $S$. mutans likely interacts and competes with a number of other oral microbial species, including commensal Streptococcus and Actinomyces species, as well as other cariogenic species $[1,5,6 \bullet, 7-9]$. Despite much of the literature focusing on interactions between bacteria, it is becoming increasingly more evident that there are medically important bacterial-fungal interactions in the oral cavity, at least in terms of mucosal infection $[10 \bullet, 11 \bullet, 12,13]$. This review discusses new evidence that suggests that interactions between $S$. mutans and $C$. albicans also occur in the presence of sucrose, which modulate the development of highly virulent biofilms on tooth surfaces, greatly influencing the pathogenesis of dental caries.

In the context of how long the field of microbiology has been studied, the window of time in which we have considered the implications of community behaviors or 
"sociomicrobiology" is very limited (>10 years) [14]. However, recently the focus has shifted to examine the complex interactions that occur among the same species, across species, and even across kingdoms during the formation of structured microbial communities termed biofilms [15-18]. The once prevalent notion that microbes are free-floating cells that operate as individuals has all but been eliminated in favor of models that examine the "social" behaviors of numerous microorganisms within biofilms [19]. Although the definition of a biofilm has fluctuated, biofilms are generally classified as a group of organisms that are adherent to a surface forming structured microenvironments/communities, which is at least in part mediated by the production of an extracellular matrix $[16,20,56]$. Biofilms range from barnacles on a ship hull to the disease-causing plaques that form on teeth and heart values $[20,21]$. Therefore, biofilms have received much attention from researchers aiming to reduce the incidence and severity of disease or costs associated with the appearance of biofilms in industrial systems. Understanding the interactions that occur between biofilm-forming organisms and the surrounding matrix environment may likely hold the key to combating and possibly eradicating these costly and often deadly biofilms.

\section{Bacterial-Fungal Interactions Are Prevalent in the Oral Cavity}

In the oral cavity, there are an estimated 700 or more species of microorganisms [22]. The diversity within the biofilm does not reflect the diversity within the saliva, as not all organisms present in the saliva are capable of adhering to pelliclecovered tooth or mucosal surfaces and/or surviving within the plaque-biofilm environment [1, 2, 4, 9, 23, 24]. However, it is apparent that dental plaque-biofilms are composed of numerous different species, and several groups have begun to investigate multispecies interactions, their metabolic activity and environmental changes within biofilms, as well as their relationship with host factors using in vitro and in vivo methods $[3,5,6 \cdot 13,25-33]$.

C. albicans is one of the most commonly detected fungal organism on human mucosal surfaces, and it often forms polymicrobial biofilms through its association with a variety of bacterial pathogens, including Escherichia coli, Pseudomonas aeruginosa, and Staphylococcus aureus [13, 17, 34-36]. These associations can enhance both fungal and bacterial virulence, ultimately amplifying disease severity and/or host mortality/morbidity. For example, C. albicans augments biofilm formation and vancomycin resistance in $S$. aureus, and while sublethal doses of either organism alone have no effect on mortality in a mouse model, co-infection results in a $100 \%$ mortality rate $[17,34]$. However, most studies have focused on the role of bacteria in enhancing Candida carriage and infectivity in mucosal diseases.

In the mouth, C. albicans is known to colonize mucosal and prosthetic surfaces and to cause tissue infection/destruction $[10 \cdot, 36-38]$. As a member of the complex oral microbiome, Candida interacts with other commensal species, namely viridans streptococci (eg, S. gordonii) [10•, 36, 39]. Tissue culture models demonstrate that $C$. albicans forms partnerships with commensal streptococci, including S. gordonii and $S$. oralis, which can enhance colonization of both organisms during oral mucosal infection [10•]. Such interactions can influence mucosal disease $[11 \bullet \cdot$. C. albicans is also associated with denture stomatitis (CADS), a set of pathological changes that occur in the oral mucosa under prostheses [37, 40-42]. The primary causes of CADS are trauma caused by the dental appliance, insufficient saliva access, and C. albicans colonization of both soft tissues and acrylic surfaces, which often occurs in the presence of $S$. gordonii $[39,40]$.

\section{C. albicans Infection Has Negative Impacts on Oral Mucosal Health}

C. albicans is a common commensal resident of the oral cavity $[37,38,43,44]$. It only becomes invasive/pathogenic when local and/or systemic predisposing factors are present. Yet, the incidence of oral candidiasis infection (including oropharyngeal and esophageal candidiasis) is increasing, which has been associated with a rise in patients that are immunocompromised [38]. Individuals frequently become immunocompromised due to HIV/AIDS infection, immunosuppressant therapy for transplant, and/or chemotherapy [38]. It is also common in patients that are effectively hyposalivated, including those with Sjogren's syndrome (autoimmune disorder of postmenopausal women) and patients that have received head and neck radiation for cancer therapy [38]. Immunocompromised patients often become chronically infected, requiring repeated topical (eg, troche) or systemic administration of antifungal agents (eg, azoles) [38]. At the same time, such infections are becoming more challenging to treat, as Candida species are steadily gaining resistance to azoles [38].

The clinical presentation of $C$. albicans on the oral mucosa may vary, but the most common forms of infection are pseudomembranous candidiasis (thrush) and erythematous candidiasis $[37,38]$. Thrush is the most widely recognized form of oral candidiasis, which tends to affect the tongue and soft palatal areas and is characterized by white plaques that may be removed with some pressure to reveal underlying erythematous tissue [37, 38]. Erythematous candidiasis is frequently associated with denture stomatitis (CADS) and refers to red atrophic areas that lack pseudomembranes [37, 38]. Erythematous candidiasis often affects the hard palate and will occur under dentures; the margin of the affected areas will 
correspond to the outline of the denture [37, 38]. Erythematous and pseudomembranous candidiasis are often asymptomatic, but either form of infection may be associated with pain and a burning or scalded sensation if the area affected is large enough or other complicating factors are present (eg, exposure to broad spectrum antibiotics) $[37,38]$. Although C. albicans is often considered the prime etiological agent of mucosal infections in these scenarios, the complex oral flora contains numerous bacterial species that can interact to augment and/or alter the progression of disease $[10 \bullet, 13,17,34-36,45,46]$.

\section{Candida-Bacterial Interactions in the Mouth}

The mere diversity of the species present within the human mouth [22], suggests that complex interactions exist between these organisms and the host that allow certain species to thrive in an environment where the resident microorganisms must compete (or synergize) for limiting or transient nutrients (from diet) and withstand salivary aggregation and clearance, as well as the host immune response [33]. Several groups have used oral mucosal biofilm models to study the ability of C. albicans to interact with other commensal species that colonize the soft tissues of the mouth, including bacteria and other fungi $[10 \bullet 11 \bullet \cdot, 12,13,47]$. Through the use of 3D tissue culture models and a mouse model, DongariBagtzoglou's group has shown that polymicrobial biofilms consisting of C. albicans and Staphylococcus, Enterococcus, and/or Lactobacillus species readily form on tongue and mucosal tissues $[10 \bullet, 11 \bullet \bullet, 13]$. More specifically, the interaction between $C$. albicans and Streptococcal species $S$. oralis, $S$. gordonii, and $S$. sanguinis enhances biofilm formation and infectivity; co-culture with $C$. albicans improves biofilm formation by these Streptococcal species, while $C$. albicans become more invasive into the mucosal tissue $[10 \bullet, 11 \bullet \cdot$. It appears that infection and invasion into the mucosal tissue by $C$. albicans elicits an immune response that involves the infiltration of immune mediators [13]. Candida has been demonstrated to induce cytokine production in the host through interactions with Dectin-1 (recognition of $\beta$-glucan) and Tolllike receptors (recognition of mannoproteins), among others $[11 \bullet, 43,48]$. $S$. oralis enhances the pathogenicity of C. albicans by strengthening the host immune response to C. albicans infection, specifically by inducing neutrophilactivating cytokines, which are more highly induced during co-infection than infection with either species alone [11••].

C. albicans interacts with various commensal Streptococcal species through at least 1 fairly specific adherence mechanism, as dissected by Jenkinson's group [39, 49, 50•]. The ability of Candida to co-adhere varies among Streptococcal species; S. gordonii, S. sanguinis, and $S$. oralis are most adherent, while $S$. salivarius and $S$. pyogenes adhere to a lesser extent, and $S$. mutans adheres poorly [49]. It was subsequently determined that the SspA and SspB ligands expressed on the surface of $S$. gordonii are involved in the interaction with C. albicans [50•]. Specifically, these adhesins bind to the Als3 adhesin expressed on the cell wall of $C$. albicans. Interruption of the coding sequence of either adhesin on S. gordonii, or the $C$. albicans adhesin, impairs binding, while expression of these adhesins on other species of fungi and bacteria (Als3 on Saccharomyces cerevisiae and $\mathrm{SspB}$ on Lactococcus lactis) is sufficient to promote an interaction [50॰]. Furthermore, Als 3 is almost exclusively expressed on the hyphal form of C. albicans, indicating that this interaction occurs specifically between $S$. gordonii cells and C. albicans hyphae. This may have an implication in pathogenesis, as hyphae are generally regarded as the more virulent/invasive form of $C$. albicans $[41,51]$. Whether or not streptococci can influence the yeast to hyphae transition in C. albicans remains to be elucidated, although it has been demonstrated in other systems that $\mathrm{Pseu}$ domonas aeruginosa can downregulate hyphae formation in its antagonist relationship with C. albicans [52].

Although S. mutans and C. albicans themselves do not coadhere well [49], available evidence does indicate that their adhesive interactions are greatly enhanced when grown in sucrose-containing media $[4,53 \bullet, 54 \cdot, 55 \bullet$. Initial observations showed that the binding of $C$. albicans and $S$. mutans to each other and to acrylic surfaces was enhanced in the presence of sucrose [53 $]$. Images from scanning electron microscopy revealed extracellular material between streptococci and yeast, suggesting that glucans may play a role in mediating binding between these organisms and/or the development of biofilms when sucrose is available [53•,54•]. Later it was demonstrated that $C$. albicans may also augment mutans streptococci biofilm formation in vitro [55•]. However, this interaction does not appear to occur in the presence of other sugars [55•], which explains earlier findings indicating that S. mutans and C. albicans largely do not interact [49].

Previous observations demonstrate that $S$. mutans-derived glucosyltransferases (Gtfs) bind to the surface of other bacteria, which can help to mediate the formation of multispecies biofilms on saliva-coated apatitic surfaces as reviewed in [4, 56]. Therefore, we hypothesized that these exoenzymes could also bind to the surface of $C$. albicans cells to produce exopolysaccharides (EPS) in situ [57••]. This could facilitate $S$. mutans-C. albicans co-adherence and the colonization of saliva-coated apatitic surfaces. We found that $\mathrm{GtfB}$ binds most effectively to $C$. albicans surfaces in an enzymatically active form, converting $C$. albicans cells into de facto glucan producers; GtfC binds less avidly than does GtfB, while GtfD binds poorly $[57 \bullet \bullet]$. Furthermore, higher quantities of glucans (with enhanced $\alpha-1,6$ linked glucose) were produced when GtfB was attached to yeast cells compared with GtfB in solution or when attached to $S$. mutans cells [57••]. Results from previous studies revealed that $\alpha-1,6$-linked glucosyl residues offer a site to which $S$. mutans binds avidly, as it 
expresses several glucan-binding proteins $[58,59]$. Thus, the formation of copious amounts of glucan on the large surface areas of yeast cells (vs bacterial cells) may provide enhanced binding sites for $S$. mutans.

We then explored the ability of $S$. mutans to bind C. albicans via a GtfB-mediated mechanism. Confocal fluorescence imaging showed large numbers of $S$. mutans cells bound to the glucans formed on the yeast cell surface [57••]. In contrast, we observed a near absence of $S$. mutans bound to uncoated $C$. albicans cells, consistent with previous findings from Jenkinson's group [49]. Thus, GtfB binding and in situ activity can convert a less receptive Candida surface into a highly adhesive site for $S$. mutans binding.

Furthermore, the presence of glucans on C. albicans cells also enhanced the fungal adhesion to saliva-coated apatitic (sHA) surfaces [57••]. We investigated whether the presence of C. albicans (with or without surface-formed glucans) could enhance $S$. mutans accumulation on sHA. We found that the presence of glucan-coated yeast cells did enhance the accumulation of $S$. mutans on sHA surfaces at least 6-fold compared with that of $S$. mutans cells incubated alone [57••]. In contrast, the presence of uncoated C. albicans cells did not increase $S$. mutans accumulation [57••], which is congruent with limited binding between the cells in the absence of sucrose/glucan [49]. Our recent work pointed to a sucrosedependent interaction that is at least partially mediated by S. mutans-derived Gtfs.

Altogether, it is clear that dynamic, specific, and pathogenesis-enhancing interactions occur between bacterial and fungal species in the oral cavity. Although much of the prior research has focused on the role of Candida-commensal bacterial interactions in soft-tissue/mucosal complications, C. albicans may also interact with the pathogen $S$. mutans in the presence of sucrose $[53 \bullet, 54 \bullet, 55 \bullet$, which may enhance infectivity, the colonization of tooth surfaces, and the pathogenesis of dental caries.

\section{C. albicans May Have a Role in the Pathogenesis of Dental Caries}

The role that $C$. albicans may play in dental caries has largely been neglected, despite clinical (microbiological) data showing that in addition to heavy infection by $S$. mutans, C. albicans is frequently detected in high numbers in plaque samples from toddlers affected by Early Childhood Caries (ECC) $[60 \bullet, 61 \bullet, 62 \bullet$. In contrast, C. albicans is either absent or detected sporadically in the plaque of healthy, ECC-free children $[60 \bullet, 61 \bullet, 62 \bullet$. Furthermore, the potential of C. albicans to induce sulcal surface (occlusal) carious lesions has been demonstrated in rats [63॰].

This area of research is of particular interest, because of its possible implication in the etiology and the pathogenesis of
$\mathrm{ECC}$, one of the most virulent, painful, and costly infectious diseases afflicting children. ECC is a hypervirulent form of dental caries that afflicts young children, which is characterized by heavy infection with $S$. mutans (often exceeding $30 \%$ of the cultivatable plaque flora) $[60 \bullet, 61 \bullet, 62 \bullet, 64-67,78]$. Children with ECC are often allowed to indulge in the protracted ingestion of dietary sugars; the child often consumes sugary beverages almost constantly from a nursing bottle [68-70], which also restricts the access of saliva to the teeth due to the mechanical effects of the nipple on the bottle. Detailed literature reviews about ECC can be found elsewhere [69-74, 78].

S. mutans has often been regarded as a key etiological agent of ECC, although other bacteria may also contribute to its pathogenesis $[60 \bullet, 61 \bullet, 62 \cdot, 64-67]$; it can convert sucrose into exopolysaccharides (EPS), the prime building blocks of cariogenic biofilms, which form the core of the extracellular matrix $[2,4,5,6 \cdot, 56]$. S. mutans glucosyltransferases (Gtfs) are constituents of the pellicle and are also adsorbed to the surface of other microorganisms, all while retaining enzymatic activity $[4,56]$. The EPS synthesized by Gtfs on these surfaces promotes local colonization and the subsequent accumulation of microbes on the teeth; at the same time, a diffusion-limiting polymeric matrix is formed that protects embedded bacteria [56]. In parallel, sucrose and other sugars are fermented by S. mutans and acidogenic organisms enmeshed in the EPSrich matrix to create acidic microenvironments within the biofilm and at the interface of attachment $[6 \bullet, 56]$. The low $\mathrm{pH}$ niches support EPS production, while cariogenic (aciduric and acidogenic) flora prosper, ensuring biofilm accretion and acid-dissolution of the adjacent tooth enamel $[56,75]$.

In ECC, overt demineralization and rampant caries rapidly and extensively occur on the free smooth surfaces of primary teeth $[71-73,78]$. However, the exact biological reasons for such a heavy infection and aggressive onset/severity of disease remain unclear. Whether the presence of Candida together with high numbers of $S$. mutans in the plaque is involved in the enhancement of biofilm virulence and in the severity of disease has been undetermined. Our recent data, combined with previous observations $[53 \bullet, 54 \bullet, 55 \bullet$, suggest strongly that $C$. albicans interactions with $S$. mutans influence the pathogenesis of ECC.

Using a combination of in vitro and in vivo approaches, we recently investigated whether $S$. mutans-C. albicans interactions play a role in co-species biofilm formation and the etiology and pathogenesis of ECC, which is described henceforth [76••]. First, we examined how the enhanced bacterialfungal interactions (mediated via Gtfs) influence biofilm formation on sHA surfaces. We focused on the development of the biofilm architecture in 3-dimensions (3D), which has been linked to the accumulation of EPS and sequential assembly of an EPS-rich matrix $[5,6 \bullet, 56]$. We used our novel approach of incorporating a fluorophore during the synthesis of 
polysaccharides by $S$. mutans-derived Gtfs, which allows us to follow the development of the matrix structure over time within intact biofilms using confocal microscopy [5, 6•].

We found that the presence of $C$. albicans along with $S$. mutans dramatically enhances the assembly of an EPSrich matrix, leading to the development of biofilms with greater biomasses compared with those formed with $S$. mutans alone (Fig. 1). Moreover, co-species biofilms accrue more EPS and harbor more viable $S$. mutans cells than singlespecies $S$. mutans biofilms. Although $C$. albicans yeast cells bind sporadically to the sHA surface in the presence of sucrose, $C$. albicans ultimately lacks the capacity to form structured biofilms under our experimental conditions.

The resulting 3D biofilm architecture differs dramatically from single-species biofilms. It is characterized by the presence of enlarged microcolonies containing $S$. mutans surrounded by both yeast and hyphal forms of C. albicans, which are all enmeshed in and surrounded by a dense and abundant EPS-rich matrix (Fig. 1). It is interesting to note that hyphae forms, which are often coated by Gtf-derived EPS, protrude from the biofilm structure into the fluid phase. Clearly, C. albicans cells may provide unique and abundant sites on both yeast and hyphal forms for EPS synthesis and accumulation within co-species biofilms. Moreover, we observed that C. albicans can also secrete matrix materials, as we detected
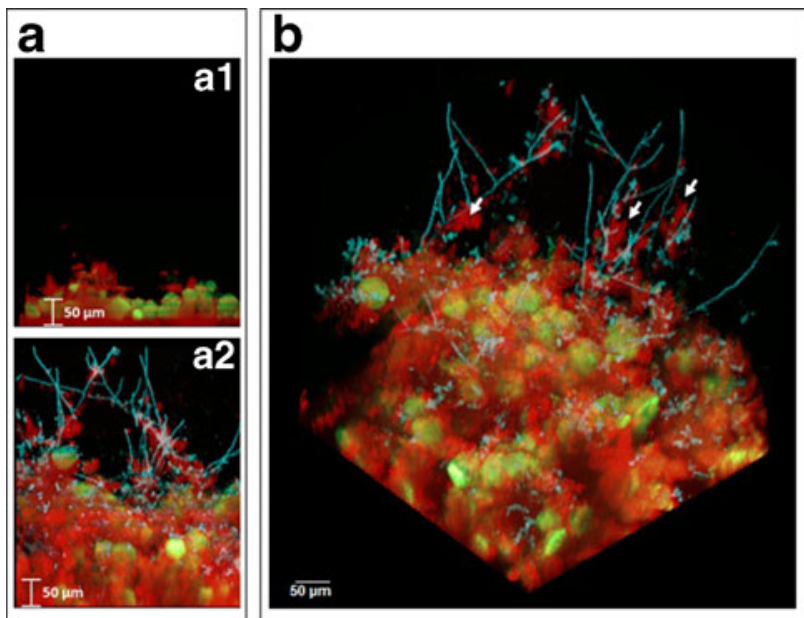

Fig. 1 Three-dimensional architecture of $S$. mutans-C. albicans co-species biofilm formed on saliva-coated hydroxyapatite. This figure has been adapted from a submitted manuscript, [76]. This figure displays representative images of (a1) single-species $S$. mutans, and (a2) co-species biofilms grown for 42 hours. Bacterial microcolonies expressing GFP are green, while fungal cells labeled with ConA-tetramethylrhodamine are blue. EPS labeled with Alexa Fluor 647 dextran is red. Panel a shows orthogonal views of the biofilms, illustrating the overall differences in the accumulation of biofilms between co-species and S. mutans UA159 single-species biofilms. Panel $\mathrm{b}$ provides a 3-D rendering of the cospecies biofilm, which illustrates the complexity of its architecture. Bacterial microcolonies and yeast forms of $C$. albicans SC5314 are enmeshed and surrounded by an EPS-rich matrix, while hyphae extend from the biofilm into the fluid-phase and are coated with EPS (denoted by white arrows) significant amounts of extracellular $\beta$-glucan interspersed with Gtf-derived EPS; $\beta$-glucan is considered to be a significant component of the extracellular matrix in biofilms formed by $C$. albicans alone on other surfaces [77].

We also examined the importance of glucan production in co-species biofilm formation by examining the structures of biofilms formed with wild type C. albicans and Gtf-deficient mutants of $S$. mutans. For example, biofilms formed with a $\Delta g t f B:$ kan $S$. mutans mutant [6 $6^{\bullet}$, showed a severe defect in biofilm formation over saliva-coated apatitic (sHA) surfaces; the populations of $S$. mutans cells in these co-species biofilms were similar to those in single-species biofilms, indicating that the lack of insoluble glucan production essentially removed the advantage of enhanced carriage conferred by cohabitation with $C$. albicans. In addition, the ability of $C$. albicans to colonize sHA surface was greatly impaired. This observation suggests that Gtf-mediated bacterial-Candida interactions are critical for the development of co-species biofilms. Collectively, our in vitro data provide a feasible explanation for the previous reports illustrating that the ability of $S$. mutans and C. albicans to form biofilms together is augmented in presence of sucrose [54•,55•]. Furthermore, it may help to explain why $S$. mutans can be found in elevated numbers along with C. albicans in the plaque of children with ECC $[60 \bullet, 61 \bullet$, 62.]. This type of interaction is a truly unique phenomenon where a bacterially-produced product adheres to and functions on the surface of an organism from another kingdom, stimulating co-existence and enhancing co-species biofilm formation.
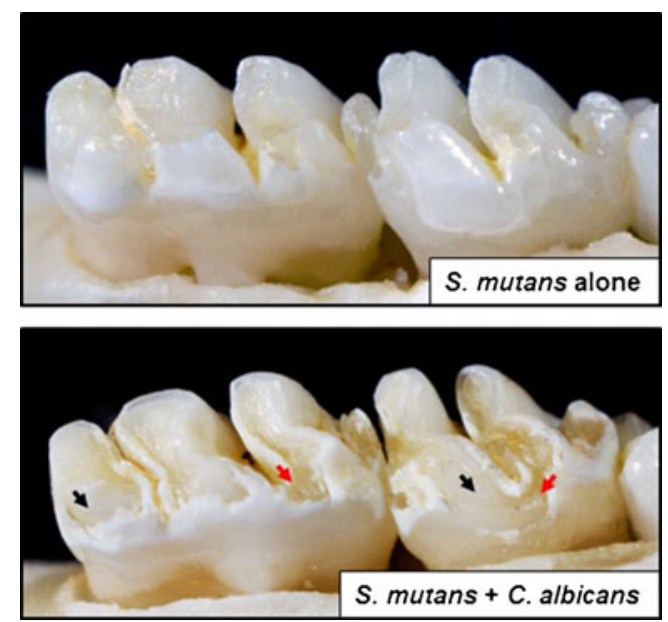

Fig. 2 Images of the teeth from rats infected with $S$. mutans UA159 alone or co-infected with C. albicans SC5314. This figure has been adapted from a submitted manuscript [76]. This figure displays photographs of lower molars in the rodent jaws; jaws representing the average result have been selected. The black arrows indicate areas of moderate to severe carious lesions in co-infected animal where areas of the enamel are missing, exposing the underlying dentin. In some areas, the dentin is eroded or missing (red arrows), denoting the most severe carious lesions. In $S$. mutans-infected animal, large areas of initial lesions were also detected, although visibly less severe than co-infected animals 
Using a rodent model that simulates the clinical conditions found in ECC, we explored the implications of this crosskingdom interaction in the pathogenesis of dental caries [76••]. The effects of co-infection with $S$. mutans and C. albicans on both the microbial colonization and the onset of caries disease in vivo were dramatic. First, we detected a significant increase in the viable populations of both $S$. mutans and C. albicans in plaque-biofilms from co-infected animals (compared with those infected with either microbe alone). Of greater significance, the virulence of plaque-biofilms in coinfected animals was synergistically enhanced, leading to the onset of severe carious lesions on the smooth surfaces of the teeth, which were characterized by large areas of exposed or missing dentin (Fig. 2). This condition clearly mirrors a major clinical feature of ECC, where extensive lesions on the free smooth surfaces of teeth are present and abundant $[69,71,73$, $74,78]$. We also observed that C. albicans induces sulcal caries, which is consistent with a previous report [63•]. Uninfected animals remained free of infection and developed lesions with negligible severity.

Altogether, our recent data [76 • $]$ provide striking evidence that $S$. mutans and $C$. albicans can develop a cooperative relationship that results in the formation of exceptionally virulent biofilms on tooth surfaces. This observation may account, at least in part, for the rapid and enhanced development of co-species biofilms and ultimately the aggressive onset of ECC.

\section{Conclusions: the Future of Understanding and Combating Oral Bacterial-Fungal Infections}

The oral cavity represents a complex environment where many species of bacteria and even fungi interact and compete to form dynamic multi-species structures on mucosal and tooth surfaces. C. albicans frequently associates with commensal oral species, which has been shown to lead to or enhance mucosal infection of the tongue and palatal tissues. Here we show evidence that $S$. mutans and $C$. albicans intimately associate with one another in the presence of sucrose via Gtf-mediated mechanism. This interaction dramatically enhances the colonization of both organisms and the development of co-species biofilms in vitro. Using an animal model, we found that oral infection with a combination of $S$. mutans and C. albicans results in enhanced infectivity and carriage of both organisms within plaque compared with animals infected with either species alone. Furthermore, the concerted actions of these organisms lead to a synergistic enhancement in biofilm virulence in vivo. The pattern of demineralization and extent of carious lesions observed in co-infected animals bears similarities to the rapid and aggressive onset of dental caries found in children with ECC. Therefore, we propose that $S$. mutans-C. albicans interactions coupled with the protracted ingestion of sucrose may ultimately enhance the development of caries in a highly susceptible population.

Altogether, previous and current findings reveal a cooperative fungal-bacterial interaction that has considerable clinical relevance, and certainly could provide new perspectives for devising efficacious therapies to control this costly and ubiquitous disease. Furthermore, the retention of $C$. albicans in biofilms associated with the tooth surfaces may also represent a reservoir of fungi that may later participate in other local or even systemic complications, as $C$. albicans alone does not seem to colonize dentition well. Naturally, further investigation is warranted to better understand the complex interactions that occur between $S$. mutans and $C$. albicans. This is an area that our group is currently devoted to studying. Understanding the dynamic nature of microbial interactions, especially those that occur across species and kingdoms, may hold the key to improved approaches to limit and remove a wide range of potentially life threating infections.

Acknowledgments Some of the work cited here has been supported by research grants from the National Institute for Dental and Craniofacial Research (T90DE021985) and from the National Science Foundation (EFRI-1137186).

\section{Compliance with Ethics Guidelines}

Conflict of Interest Dr. Megan Falsetta received a grant from NIH/ NIDCR. Dr. Hyun Koo received grants from NIH and NSF. Dr. Koo serves as a section editor for Current Oral Health Reports.

Human and Animal Rights and Informed Consent This article does not contain any studies with human or animal subjects performed by any of the authors.

\section{References}

Papers of particular interest, published recently, have been highlighted as:

- Of importance

•. Of major importance

1. Marsh PD. Are dental diseases examples of ecological catastrophes? Microbiology. 2003;149(Pt 2):279-94.

2. Paes Leme AF, Koo H, Bellato CM et al. The role of sucrose in cariogenic dental biofilm formation - new insight. J Dent Res. 2006;85:878-87.

3. Nyvad B, Crielaard W, Mira A et al. Dental caries from a molecular microbiological perspective. Caries Res. 2013;47:89-102.

4. Bowen WH, Koo H. Biology of Streptococcus mutans-Derived Glucosyltransferases: role in extracellular matrix formation of cariogenic biofilms. Caries Res. 2011;45:69-86.

5. Koo H, Xiao J, Klein MI et al. Exopolysaccharides produced by Streptococcus mutans glucosyltransferases modulate the establishment of microcolonies within multispecies biofilms. J Bacteriol. 2010;192:3024-32.

6. Xiao J, Klein MI, Falsetta ML et al. The exopolysaccharide matrix modulates the interaction between $3 \mathrm{D}$ architecture and 
virulence of a mixed-species oral biofilm. PLoS Pathog. 2012;8:e1002623. This paper demonstrates the critical role of $S$. mutans glucosyltransferase-derived glucans in the assembly of a $3 D$ biofilm matrix-scaffold and low pH microenvironments in the presence of other oral bacterial species.

7. Kreth J, Merritt J, Shi W et al. Competition and coexistence between Streptococcus mutans and Streptococcus sanguinis in the dental biofilm. J Bacteriol. 2005;187:7193-203.

8. Beighton D. The complex oral microflora of high-risk individuals and groups and its role in the caries process. Community Dent Oral Epidemiol. 2005;33:248-55.

9. Takahashi N, Nyvad B. The role of bacteria in the caries process: ecological perspectives. J Dent Res. 2011;90:294-303.

10. Diaz PI, Xie Z, Sobue T et al. Synergistic interaction between Candida albicans and commensal oral streptococci in a novel in vitro mucosal model. Infect Immun. 2012;80:620-32. This paper demonstrates that $C$. albicans and oral streptococci may augment each other's virulence using a novel in vitro model.

11.• Xu H, Sobue T, Thompson A et al. Streptococcal co-infection augments Candida pathogenicity by amplifying the mucosal inflammatory response. Cell Microbiol. 2013. doi:10.111/cmi.12216 This paper demonstrates one key mechanism by which streptococcal species may augment fungal virulence in vivo.

12. Silva S, Henriques M, Hayes A et al. Candida glabrata and Candida albicans co-infection of an in vitro oral epithelium. J Oral Pathol Med. 2011;40:421-7.

13. Dongari-Bagtzoglou A, Kashleva H, Dwivedi P et al. Characterization of mucosal Candida albicans biofilms. PLoS One. 2009;4:e7967.

14. Parsek MR, Greenberg EP. Sociomicrobiology: the connections between quorum sensing and biofilms. Trends Microbiol. 2005; 13:27-33.

15. Hall-Stoodley L, Costerton JW, Stoodley P. Bacterial biofilms: from the natural environment to infectious diseases. Nat Rev Microbiol. 2004;2:95-108.

16. Flemming HC, Wingender J. The biofilm matrix. Nat Rev Microbiol. 2010;8:623-33.

17. Shirtliff ME, Peters BM, Jabra-Rizk MA. Cross-kingdom interactions: Candida albicans and bacteria. FEMS Microbiol Lett. 2009;299:1-8.

18. Davey ME, O'Toole G A. Microbial biofilms: from ecology to molecular genetics. Microbiol Mol Biol Rev. 2000;64:847-67.

19. Donlan RM, Costerton JW. Biofilms: survival mechanisms of clinically relevant microorganisms. Clin Microbiol Rev. 2002;15: 167-93.

20. Costerton JW. Introduction to biofilm. Int J Antimicrob Agents. 1999;11:217-21. discussion 237-9.

21. Dunne Jr WM. Bacterial adhesion: seen any good biofilms lately? Clin Microbiol Rev. 2002;15:155-66.

22. Dewhirst FE, Chen T, Izard J et al. The human oral microbiome. J Bacteriol. 2010;192:5002-17.

23. Nobbs AH, Jenkinson HF, Jakubovics NS. Stick to your gums: mechanisms of oral microbial adherence. J Dent Res. 2011;90: 1271-8.

24. Nobbs AH, Lamont RJ, Jenkinson HF. Streptococcus adherence and colonization. Microbiol Mol Biol Rev. 2009;73:407-50. Table of Contents.

25. Guggenheim B, Giertsen E, Schupbach P et al. Validation of an in vitro biofilm model of supragingival plaque. J Dent Res. 2001;80:363-70

26. Reese S, Guggenheim B. A novel TEM contrasting technique for extracellular polysaccharides in vitro biofilms. Microsc Res Tech. 2007;70:816-22.

27. Wood SR, Kirkham J, Marsh PD et al. Architecture of intact natural human plaque biofilms studied by confocal laser scanning microscopy. J Dent Res. 2000;79:21-7.
28. Palmer Jr RJ. Supragingival and subgingival plaque: paradigm of biofilms. Compend Contin Educ Dent. 2010;31:104-6. 108, 110 passim; quiz 124, 138.

29. Kreth J, Zhang Y, Herzberg MC. Streptococcal antagonism in oral biofilms: Streptococcus sanguinis and Streptococcus gordonii interference with Streptococcus mutans. J Bacteriol. 2008;190: 4632-40.

30. Qi F, Kreth J. Characterization of anti-competitor activities produced by oral bacteria. Methods Mol Biol. 2010;666:151-66.

31. Merritt J, Qi F. The mutacins of Streptococcus mutans: regulation and ecology. Mol Oral Microbiol. 2012;27:57-69.

32. Wang Q, Wright CJ, Dingming $\mathrm{H}$ et al. Oral community interactions of filifactor alocis in vitro. PLoS One. 2013;8:e76271.

33. Jenkinson HF. Beyond the oral microbiome. Environ Microbiol. 2011;13:3077-87.

34. Harriott MM, Noverr MC. Importance of Candida-bacterial polymicrobial biofilms in disease. Trends Microbiol. 2011;19: 557-63.

35. Peleg AY, Hogan DA, Mylonakis E. Medically important bacterial-fungal interactions. Nat Rev Microbiol. 2010;8: $340-9$.

36. Thein ZM, Seneviratne CJ, Samaranayake YH et al. Community lifestyle of Candida in mixed biofilms: a mini-review. Mycoses. 2009;52:467-75.

37. Lalla RV, Patton LL, Dongari-Bagtzoglou A. Oral candidiasis: pathogenesis, clinical presentation, diagnosis and treatment strategies. J Calif Dent Assoc. 2013;41:263-8.

38. Samaranayake LP, Keung Leung W, Jin L. Oral mucosal fungal infections. Periodontol 2000. 2009;49:39-59.

39. Jenkinson HF, Douglas LJ. Candida interactions with bacterial biofilms. In: Brogden KA, Guthmiller JM, editors. Polymicrobial infections and disease. Washington, DC: ASM Press; 2002. p. 35773.

40. Ganguly S, Mitchell AP. Mucosal biofilms of Candida albicans. Curr Opin Microbiol. 2011;14:380-5.

41. Dongari-Bagtzoglou A. Mucosal biofilms: challenges and future directions. Expert Rev Anti Infect Ther. 2008;6:141-4.

42. Ellepola AN, Samaranayake LP. Oral candidal infections and antimycotics. Crit Rev Oral Biol Med. 2000;11:172-98.

43. Moyes DL, Naglik JR. Mucosal immunity and Candida albicans infection. Clin Dev Immunol. 2011;2011:346307.

44. Sardi JC, Almeida AM, Mendes Giannini MJ. New antimicrobial therapies used against fungi present in subgingival sites - a brief review. Arch Oral Biol. 2011;56:951-9.

45. Nobile CJ, Fox EP, Nett JE et al. A recently evolved transcriptional network controls biofilm development in Candida albicans. Cell. 2012;148:126-38.

46. Morales DK, Hogan DA. Candida albicans interactions with bacteria in the context of human health and disease. PLoS Pathog. 2010;6:e1000886

47. Peters BM, Ovchinnikova ES, Krom BP et al. Staphylococcus aureus adherence to Candida albicans hyphae is mediated by the hyphal adhesin Als3p. Microbiology. 2012;158(Pt 12): 2975-86.

48. Gow NA, van de Veerdonk FL, Brown AJ et al. Candida albicans morphogenesis and host defence: discriminating invasion from colonization. Nat Rev Microbiol. 2012;10:112-22.

49. Jenkinson HF, Lala HC, Shepherd MG. Coaggregation of streptococcus sanguis and other streptococci with Candida albicans. Infect Immun. 1990;58:1429-36.

50. Silverman RJ, Nobbs AH, Vickerman MM et al. Interaction of Candida albicans cell wall Als3 protein with streptococcus gordonii $\mathrm{SspB}$ adhesin promotes development of mixed-species communities. Infect Immun. 2010;78:4644-52. This paper describes at least one specific co-adherence mechanism for fungal and bacterial species, C. albicans and S. gordonii. 
51. Blankenship JR, Mitchell AP. How to build a biofilm: a fungal perspective. Curr Opin Microbiol. 2006;9:588-94.

52. Bandara HM, K Cheung BP, Watt RM et al. Pseudomonas aeruginosa lipopolysaccharide inhibits Candida albicans hyphae formation and alters gene expression during biofilm development. Mol Oral Microbiol. 2013;28:54-69.

53. Branting C, Sund ML, Linder LE. The influence of Streptococcus mutans on adhesion of Candida albicans to acrylic surfaces in vitro. Arch Oral Biol. 1989;34:347-53. This is the first study demonstrating that S. mutans and C. albicans interact and co-adhere in the presence of sucrose.

54. Metwalli KH, Khan SA, Krom BP et al. Streptococcus mutans, Candida albicans, and the human mouth: a sticky situation. PLoS Pathog. 2013;9:e1003616. This report emphasizes the need for further research to elucidate the mechanisms and the in vivo implications of S. mutans and C. albicans interactions and co-existence in biofilms.

55. Pereira-Cenci T, Deng DM, Kraneveld EA et al. The effect of Streptococcus mutans and Candida glabrata on Candida albicans biofilms formed on different surfaces. Arch Oral Biol. 2008;53: 755-64. This paper was one of the first studies showing that $C$. albicans can influence biofilm formation by S. mutans in the presence of sucrose.

56. Koo H, Falsetta ML, Klein MI. The exopolysaccharide matrix: a virulence determinant of cariogenic biofilm. J Dent Res. 2013;92: $1065-73$.

57.• Gregoire S, Xiao J, Silva BB et al. Role of glucosyltransferase B in the interactions of Candida albicans with Streptococcus mutans and experimental pellicle formed on hydroxyapatite surface. Appl Environ Microbiol. 2011;77:6357. This study reveals a key mechanism for the observed sucrose-dependent adhesive interaction between S. mutans and C. albicans, which was found to be largely mediated by localized glucan production via streptococcal Gtfs attached to the fungal surface.

58. Schilling KM, Bowen WH. Glucans synthesized in situ in experimental salivary pellicle function as specific binding sites for Streptococcus mutans. Infect Immun. 1992;60:284-95.

59. Banas JA, Vickerman MM. Glucan-binding proteins of the oral streptococci. Crit Rev Oral Biol Med. 2003;14:89-99.

$60 . \bullet$ de Carvalho FG, Silva DS, Hebling J et al. Presence of mutans streptococci and Candida spp. in dental plaque/dentine of carious teeth and early childhood caries. Arch Oral Biol. 2006;51:1024-8. This is one of the first clinical studies that clearly show that $C$. albicans is present in the plaque and saliva of children with ECC, along with elevated numbers of $S$. mutans.

61. Raja M, Hannan A, Ali K. Association of oral candidal carriage with dental caries in children. Caries Res. 2010;44:272-6. This paper also demonstrates that $C$. albicans is found in children with ECC.

62. Yang XQ, Zeng X, Ning K et al. Saliva microbiomes distinguish caries-active from healthy. human populations. ISME J. 2012;6:110. This study also demonstrates that $C$. albicans is found in children with ECC.
63. Klinke T, Guggenheim B, Klimm W et al. Dental caries in rats associated with Candida albicans. Caries Res. 2011;45:100-6. This paper shows that C. albicans is capable of causing sulcal surface carious lesions in rats.

64. Berkowitz RJ, Koo H, McDermott MP et al. Adjunctive chemotherapeutic suppression of mutans streptococci in the setting of severe early childhood caries: an exploratory study. J Public Health Dent. 2009;69:163-7.

65. Kanasi E, Dewhirst FE, Chalmers NI et al. Clonal analysis of the microbiota of severe early childhood caries. Caries Res. 2010;44: 485-97.

66. Hughes CV, Dahlan M, Papadopolou E et al. Aciduric microbiota and mutans streptococci in severe and recurrent severe early childhood caries. Pediatr Dent. 2012;34:e16-23.

67. Becker MR, Paster BJ, Leys EJ et al. Molecular analysis of bacterial species associated with childhood caries. J Clin Microbiol. 2002;40:1001-9.

68. Palmer CA, Kent R Jr, Loo CY et al. Diet and caries-associated bacteria in severe early childhood caries. J Dent Res. 2010;89: 1224-9.

69. Chestnutt IG, Murdoch C, Robson KF. Parents and carers' choice of drinks for infants and toddlers, in areas of social and economic disadvantage. Community Dent Health. 2003;20:139-45.

70. Mobley C, Marshall TA, Milgrom P et al. The contribution of dietary factors to dental caries and disparities in caries. Acad Pediatr. 2009;9:410-4.

71. Dye BA, Tan S, Smith V et al. Trends in oral health status: United States, 1988-1994 and 1999-2004. Vital Health Stat. 2007;11(248): $1-92$.

72. Tinanoff N, Reisine S. Update on early childhood caries since the Surgeon General's Report. Acad Pediatr. 2009;9:396-403.

73. Casamassimo PS, Thikkurissy S, Edelstein BL et al. Beyond the DMFT: the human and economic cost of early childhood caries. J Am Dent Assoc. 2009;140:650-7.

74. Chou R, Cantor A, Zakher B et al. Preventing dental caries in children $<5$ years: systematic review updating USPSTF recommendation. Pediatrics. 2013;132:332-50.

75. Lemos JA, Burne RA. A model of efficiency: stress tolerance by Streptococcus mutans. Microbiology. 2008;154(Pt 11):3247-55.

76.• Falsetta ML, Klein MI, Colonne PM et al. Symbiotic relationship between Streptococcus mutans and Candida albicans synergizes the virulence of plaque-biofilms in vivo. PLoS Pathog. [Submitted December, 2013]. This paper provides striking in vivo evidence that co-infection with $S$. mutans and C. albicans synergistically enhance the onset and severity of dental caries using a rodent model that simulates the clinical conditions of ECC as well as possible mechanisms of interaction.

77. Taff HT, Nett JE, Zarnowski R et al. A Candida biofilm-induced pathway for matrix glucan delivery: implications for drug resistance. PLoS Pathog. 2012;8:e1002848.

78. Karp J, Berkowitz RJ. Clincal outcomes for severe early childhood caries. Clin Rev Pediatr. 2008;4:169-73. 